\title{
L'organisation des S.H.S. en écoles d'ingénieurs, deux exemples opposés
}

\author{
Michel Cotte ${ }^{\mathrm{a}}$ \\ Centre François Viète, Université de Nantes, France
}

\begin{abstract}
Résumé. Nous poursuivons ici la réflexion générale abordée dans l'article de cette même publication : "Les SHS en écoles d'ingénieurs, entre utilité pratique et formation culturelle », par la présentation d'études de cas. Il s'agit de deux exemples de fonctionnement ou de mise en place d'enseignements répondant à l'appellation très générale de sciences humaines et sociales en écoles d'ingénieurs, au cours des années 19902000. Ils illustrent pour nous convenablement les deux pôles conceptuels proposés afin de comprendre la problématique des SHS en écoles d'ingénieurs : l'une est composée de départements de spécialisés accolés (Polytech Nantes) et l'autre affiche une vocation généraliste avec une centralisation pédagogique plus marquée (l'Université de technologie de Belfort - Montbéliard).
\end{abstract}

\begin{abstract}
There we pursue the general thought started in former paper of this publication: "Human and Social Sciences in Engineering Schools: useful teaching versus cultural education", by presenting some case studies. They are two examples of functioning or setting of human and social sciences programs, in large sense, inside engineering schools along the 90s and 2000s. In our view, they satisfyingly illustrate the two conceptual poles already evocated for understand the status of human and social sciences into engineering schools: one is a group of parallel specialized departments (Polytech Nantes); the other claims its generalist educational purpose with a more centralized pedagogical organization (University of Technology of Belfort-Montbéliard).
\end{abstract}

Dans la conclusion d'un premier article publié dans ce volume, Michel Cotte, « Les SHS en écoles d'ingénieurs, entre utilité pratique et formation culturelle » (Cotte 2014), nous avons souligné l'enjeu organisationnel essentiel des sciences humaines et sociales (SHS) en école d'ingénieurs, au sein d'un espace horaire contraint mais conséquent. Nous avons noté que cela était dû notamment à l'influence de la Commission du titre d'ingénieur $(\mathrm{CTI})^{1}$, mais avec des consignes relativement vagues qu'il appartient aux écoles d'interpréter et de mettre en pratique suivant leur projet pédagogique général. Cet espace horaire est à l'origine d'un véritable rapport de force entre direction d'école, ou de département, et le groupe des collègues concernés, mais un rapport de force qui peut être productif et constructif, comme nous allons le voir ici au sein de deux exemples bien différents.

\footnotetext{
a e-mail : michel.cotte@univ-nantes.fr

${ }^{1}$ La CTI est l'organisme habilité, en France, à accréditer le diplôme d'ingénieur délivré par les écoles.
}

This is an Open Access article distributed under the terms of the Creative Commons Attribution License 4.0, which permits unrestricted use, distribution, and reproduction in any medium, provided the original work is properly cited. 
Nous avons également vu dans ce premier article, que le terme de sciences humaines et sociales était fréquemment utilisé en écoles d'ingénieurs, mais dans un sens assez large, voire carrément flou. Cette appellation joue le rôle d'un terme générique, dont le contenu réel change d'une école à l'autre en fonction de ses objectifs propres, et aussi suivant ses utilisateurs au sein d'une même école. Elle entretient un rapport parfois lointain avec les champs académiques classiquement identifiés comme des sciences humaines et sociales. Il y a bien entendu un questionnement qui apparaît à ce sujet et que nous avons noté : s'agit-il de morceaux choisis des SHS classiques en relation avec le monde des ingénieurs et de l'entreprise ?, ou bien de branches spécifiques des SHS développées par des problématiques propres à ces systèmes sociotechniques?, ou encore de SHS « appliquées » comme il existe des sciences exactes appliquées, justement à une série de questions technologiques et industrielles !

Le terme « Humanités » est également utilisé dans notre propos, dans un sens voisin mais encore plus large et plus englobant de tout ce qui n'est pas directement un enseignement scientifique ou technologique. Il s'agit là d'une enveloppe dont les contenus sont variables d'une école à une autre et qui correspond en définitive à une maquette pédagogique, comme nous allons le voir dans deux études de cas. Notre usage de ces termes sera donc proche, et il suivra d'une part les contextes socioprofessionnels de leur usage («Humanités » est caractéristique par exemple du vocabulaire de l'Université de technologie de Belfort-Montbéliard où il a en outre un sens administratif), d'autre part une nuance entre des contenus relativement bien identifiés et légitimés pour les sciences humaines et sociales et une approche plus globale et inclusive de matières enseignées très diverses (langues, communication, pratiques sportives...) pour celui d'Humanités.

Dans notre premier article, nous avons également mis en évidence, le rôle essentiel joué par les deux grands types d'écoles d'ingénieurs sur les contenus des enseignements de SHS en leur sein. Il s'agit dans ce second article de développer deux exemples précis, à partir de notre expérience personnelle d'enseignant en histoire des techniques en écoles d'ingénieurs puis en tant que directeur de département ou de service de sciences humaines et sociales en école ${ }^{2}$. Une telle expérience montre la réalité du terrain, ses spécificités, et nous rappelle que pour jouer sur les mécanismes de décisions pédagogiques, il faut accepter une approche générale de ces enseignements plutôt qu'une entrée par somme de disciplines indépendantes. En d'autres termes, essayer de poser la question des sciences humaines et sociales en école d'ingénieur à partir d'une question pédagogique précise et épistémologiquement bien déterminée, comme l'histoire des sciences, risque de nous faire passer à côté d'une intelligence du phénomène « école », vu comme un ensemble pédagogique finalisé et fortement structuré. C'est cette approche globale des sciences humaines et sociales en écoles, que nous prenons ici en assumant la difficulté à correctement définir ces champs de connaissances comme leurs problématiques propres.

Dans ces diverses perspectives, nous présentons ici deux études de cas à caractère global : la mise en place du service transversal HES (Homme, Entreprise et Société) à Polytech Nantes, au milieu des années 2000, puis le fonctionnement du département des « Humanités » à l'Université de technologie de Belfort-Montbéliard, à la fin des années 1990. La première illustre le devenir des sciences humaines et sociales dans une école en cours de constitution, par agrégation de différentes branches de formations spécialisées d'ingénieurs avec leur histoire propre et des sensibilités variables des acteurs aux différents domaines des sciences humaines et sociales. La seconde part d'un point de vue généraliste dans la définition de la formation des élèves ingénieurs, a priori beaucoup plus ouvert sur les Humanités et bénéficiant d'une direction centrale effective et de services pédagogiques transversaux aux diverses filières de formation technologiques.

\footnotetext{
${ }^{2}$ La majeure partie de mes activités universitaires s'est exercée en école d'ingénieurs, en tant qu'historien des techniques. J'ai été successivement et parfois parallèlement, entre la fin des années 1980 et 2007, en charge de cours à l'ENTPE à Vaulx-en-Velin, à l'UT de Belfort-Montbéliard, à l'Ecole polytechnique de l'Université de Nantes, et à l'Ecole Centrale de Nantes. J'ai également dirigé le département des Humanités de l'UTBM (1998-2003) et le département Homme Entreprise et Société à Polytech Nantes (2004-2007).
} 


\section{Polytech nantes et le service transversal H.E.S. (homme, entreprise et société)}

\subsection{Contexte général des écoles d'ingénieur Polytechs, Polytech Nantes}

Les écoles «polytech » (écoles polytechniques au sein des universités françaises), déjà évoquées dans notre premier article, se sont développées à partir de la fin des années 1980 et surtout dans les années 1990, sur l'idée initiatrice de conserver de les développer au sein des universités des compétences pédagogiques de sciences appliquées. Ces initiatives s'appuyèrent souvent sur de bonnes équipes locales de recherches, comme à Grenoble où à Nantes ${ }^{3}$. L'une des idées était aussi d'offrir un premier cycle préparatoire via les anciens DEUG, aujourd'hui les deux premières années de licence, à des étudiants de bon niveau scientifique mais qui, dans le système classiques des « taupes » et des concours, n'avaient guère l'opportunité d'accéder à des écoles d'ingénieurs. Adroitement, les polytechs se sont situés sur tous les créneaux du recrutement pour leurs élèves, sans en privilégier aucun : outre les premiers cycles universitaires adaptés, on y retrouve de nombreux élèves de prépas ${ }^{4}$ n'ayant pas obtenu les concours visés ou soucieux de s'ancrer dans une région donnée, et des «têtes de promos » tant de DUT que de BTS $^{5}$. Dans les années 2000, le réseau des polytechs s'est rapidement étoffé pour devenir présent dans plus d'une douzaine d'universités, dans toute la France y compris à Paris. Quantitativement, c'est aujourd'hui le premier réseau français d'écoles, avec celui des ENSI. Dernier venu, il semble avoir réussi là où les projets antérieurs de rénovation des filières de formation des ingénieurs, souvent assez ambitieux dans leur affichage initial (INSA puis UT), ont assez vite plafonné en termes de réseau, après d'incontestables succès initiaux. Les coûts-élèves de ces différentes écoles seraient aussi à considérer pour expliquer leurs succès ou insuccès institutionnels en période de rigueur budgétaire...

Le pragmatisme a prévalu, avec un effort certain de professionnalisation de la part des universitaires scientifiques ayant pris en charge ces projets de formation. La compétence métier y est devenue quasiment un mythe et une véritable identité. Pour un certain nombre d'observateurs professionnels et académiques, dans la continuité de la création des IUT une vingtaine d'années auparavant, l'université rencontrait le monde de l'entreprise et travaillait avec lui à la formation de ses cadres techniques. La création d'écoles par addition de départements fortement autonomes, liés à une équipe pédagogique fondatrice, fut son mode d'organisation privilégié, garant d'une bonne lisibilité professionnelle. En pratique, ce fut également un outil efficace d'absorption de petites écoles d'ingénieurs déjà en place et répondant à des besoins locaux spécifiques, à l'initiative de pouvoirs locaux du type Chambre de commerce. Celles-ci, loin de pouvoir atteindre une taille critique par elles-mêmes, étaient souvent en mal d'un rattachement de type fédéral qui ne remettrait pas trop en cause tant leur identité initiale que leurs modes pédagogiques. Par exemple, l'école d'ingénieurs de Saint-Nazaire, créée pour les besoins en électrotechniciens de la construction navale, devenait, en douceur, le département de Génie électrique de Polytech Nantes, comme $5^{\text {ème }}$ département de la fratrie. Ce dernier venu formait simplement une annexe délocalisée du double vaisseau amiral, construit sur le technopôle de Nantes, Atlantech, dans les années 90 et qui correspondait déjà à l'union de deux écoles voisines, l'une orientée matériaux et énergie, l'autre électronique et informatique, à l'origine de Polytech Nantes. Chacun gardait ses spécialités, ses profs, ses locaux et ses intervenants professionnels pour former cinq départements dont «GE » à Saint-Nazaire (2007).

Il s'agit donc d'un type d'école où les départements sont à l'origine même de l'école, dont la création et la croissance externe sont essentiellement vécues comme une économie d'échelle à caractère

\footnotetext{
${ }^{3}$ Dans l'Après-guerre, la création des ENSI avait envisagé un projet similaire pour les universités de provinces mais, bien vite, la logique «école d'ingénieur » des 30 glorieuses les a séparées de l'université pour en faire des écoles autonomes.

4 Classes préparatoires.

${ }^{5}$ DUT Diplome universitaires de technologies, formation en deux ans dispensées par les universités et Brevet de technicien supérieur, formation en deux ans post-bac dispensées par les lycées techniques.
} 
administratif et pour une meilleure lisibilité extérieure (recrutement, relation avec les pouvoirs publics, les entreprises... ). Les départements sont puissamment organisés, en particulier sur le plan des grilles horaires et des emplois du temps. Une forte concertation entre le enseignants de science et de technologie existe depuis les origines au sein des départements, conduisant à des équilibres subtils entre les diverses disciplines et tenant compte des obligations statutaires de chaque collège du département, des rapports de force internes, du poids des laboratoires rattachés, etc. Ces grilles annuelles horizontales, par année de formation, ont par la suite été confrontées à des velléités de stratégie fédérale, au fur et à mesure de l'extension de l'école, mais principalement pour des tâches d'accompagnement et d'environnement. Celles-ci relèvent d'ailleurs plus de logiques de site que d'une école à proprement parler : gestion technique des bâtiments, horaires d'ouverture et gardiennage, service informatique école, service de communication, des relations industrielles, etc. L'aspect réellement commun à l'ensemble de l'école se limite au départ à une gestion administrative centralisée autour d'un directeur et de services généraux.

Le besoin d'enseignements des SHS, au sens large, suscita lui aussi, à l'origine, une approche au sein des départements : avoir son enseignant de langue, son référent en écogestion, son animateur sportif capable de concrétiser, comme un coach de club, une bonne image du département dans son environnement étudiant. La montée en puissance de l'école était surtout une opportunité de mutualiser les moyens pédagogiques non scientifiques, en fidélisant par des postes permanents ceux qui s'étaient totalement moulés dans cette logique initiale de branche professionnelle. Les premières mises en place d'enseignements de ce type, dans les années 90, suivaient une définition partant exclusivement des besoins des départements, besoins d'abord prescrits par les enseignants scientifiques et technologiques, et conçus en complément directs de leurs propres formations comme de l'idée qu'ils se faisaient du monde industriel dans leur spécialité technologique.

La pression de la CTI $^{6}$ s'est d'abord exercée sur l'anglais qui, incontestablement, posait un problème pour beaucoup d'élèves d'une école de ce type, notamment ceux passés par les prépas intégrées de l'université ou les BTS. Une grande diversité de niveau était en général la règle et des méthodes volontaristes ont été, en effet, d'une certaine efficacité. Elle s'est ensuite exercée par les recommandations sur les horaires et, à un degré moindre, par des consignes lancées un peu comme on lance une mode, par exemple l'impérieux besoin de faire de l' « éthique » en école d'ingénieurs, à la fin des années 90 (Didier 2008), sans que grand monde ne sache vraiment de quoi il s'agissait...la consigne était alors que le mot devait absolument apparaître sur une grille horaire!

La volonté de certains directeurs d'écoles, à certain moments clés de l'histoire d'une école, joue également dans certaines orientations générales. Par exemple, le projet d'intégration de l'IHT (Institut de l'Homme et de la Technologie) à Polytech Nantes, alors dirigé par Bernard Remaud, dans le début des années 2000, a été l'un de ces moments. Ce fut le motif de mon affectation personnelle dans cette école, via la présidence de l'Université de Nantes. Cet institut, à caractère simultanément universitaire et régional, s'était développé de manière autonome. Après une première période ambitieuse de noyau séminal devant permettre la création d'une Université de technologie, il était devenu un service transversal du technopôle nantais d'Atlantech qui comprenait une bibliothèque technologique, un centre de conférences, un support de projets de recherche et développement comme le pôle du patrimoine scientifique, etc.. Il s'était essentiellement développé comme centre de formation continue aux risques et à la sécurité. Il disposait également de quelques chercheurs isolés, parfois brillants, notamment en sociologie et dans le domaine des risques. J'apportais moi-même mes compétences en histoire des techniques avec le projet des maquettes numériques de machines anciennes ${ }^{7}$.

\footnotetext{
6 Voir le premier article, de l'auteur de ce volume, pour le rôle de cet organisme d'accréditation du diplôme d'ingénieur.

${ }^{7}$ Ce projet de recherche s'est développé sous le nom de pôle OSTIC (Objet, Société et TIC) entre 2004 et 2007.
} 
Les sciences humaines dans les parcours scientifiques et techniques professionnalisants

Tableau 1. L'équipe HES - IHT en charge de la formation aux SHS à Polytech Nantes (rentrée 2006).

\begin{tabular}{|ll|}
\hline Enseignants - chercheurs titulaires : & 3 (dont 2 IHT) \\
PRAG: & 3 \\
Associés plein temps : & 2 (IHT) \\
PAST mi-temps : & 2 (IHT) \\
Chargé de mission formation permanente : & 1 (IHT) \\
Intervenant de l'école hors HES-IHT (temps partiel) & 1 (Relations \\
& \multicolumn{1}{c}{ industrielles) } \\
Un portefeuille diversifié de vacataires : & $7-8$ \\
$\quad$ académiques & $8-10$ \\
Secrétaire: $\quad$ professionnels & 1 \\
\hline
\end{tabular}

\subsection{La mise en place d'une équipe de SHS pour l'école et ses projets initiaux}

En parallèle d'une histoire complexe de l'intégration de l'Institut de l'homme et de la technologie (IHT) à l'Université de Nantes, due notamment à un changement trop rapide de l'équipe de direction de Polytech à la rentrée 2005, l'une des missions initiale fut d'intégrer les personnels de l'IHT et ceux déjà en poste dans l'école, hors service des langues ${ }^{8}$. Sur un plan pédagogique, il s'agissait de restructurer le service HES (Homme, entreprise et société) de l'école déjà existant, en particulier d'en redéfinir la maquette pédagogique en lien avec la mise en place d'une équipe nouvelle. En outre, cela coïncidait avec une remise à plat de tous les enseignements en lien avec d'une part avec l'évaluation en préparation par la CTI et d'autre part le franchissement d'un cap dans l'intégration au niveau école avec l'arrivée du département GE de Saint-Nazaire. L'équipe pédagogique dite alors HES-IHT regroupait un ensemble relativement réduit, mais tout de même significatif pour une école qui approchait 300 diplômes par an (Tableau 1). Elle comprenait une douzaine de personnels permanents, dont trois à temps partiel. Sur le fond historique du service HES (sciences de gestion, économie, relations industrielles, EPS) venait se greffer les ressources propres en enseignants - chercheurs de l'IHT (sociologie, risque industriel, qualité, organisation du travail, histoire des techniques, patrimoine industriel) ainsi que le recrutement d'un maître de conférences (sciences de gestion).

La stratégie de l'ensemble HES-IHT allait s'exercer en direction de l'école et en direction des départements. Il fallait d'abord asseoir la présence des sciences humaines et sociales dans l'école, avec une stratégie initiale qui visait à le transformer en département à part entière. Le fait de bénéficier de locaux en propre, ceux de l'IHT historique $\left(1500 \mathrm{~m}^{2}\right)$, avec des salles de cours et de conférences, enfin de disposer de reliquats appréciables de subventions autorisait une certaine ambition. En accord avec la direction du départ du projet, un département «Ingénieur des collectivités territoriales » était esquissé, où les départements classiques apportaient une large gamme de savoir-faire (énergétique, électricité courants forts, matériaux et informatique réseaux), ainsi que du génie civil et du bâtiment en compétences extérieures proches. Les SHS assuraient de leur côté une part importante de la formation et la coordination de l'ensemble. L'ambition de devenir un département à part entière, dans une école de spécialistes, était justifiée. Malheureusement, le changement brutal de direction entraina l'arrêt du projet et le siphonage des derniers subsides de l'Institut... Il s'agissait d'un échec évident, clairement lié au changement de direction et d'orientation future de l'école, mais peut-être aussi à un manque

\footnotetext{
${ }^{8}$ L'intégration ou pas des langues aux SHS est une situation variable suivant les écoles, leurs traditions et le type d'enseignants y exerçant. Lorsqu'il existe un département SHS fortement organisé, elles sont généralement intégrées à cet ensemble, notamment pour des économies de moyens.
} 
d'approfondissement interne du projet par l'équipe mise en place pour cela. Il en resta toutefois la création d'une option transversale de $5^{\mathrm{e}}$ année ( $3^{\mathrm{e}}$ année ingénieur), permettant le stage de fin d'études sous la responsabilité HES-IHT : l'option transversale «Management des organisations et des risques industriels » (MORI). Cette option commune à tous les départements connut un succès important auprès des élèves. Certain départements allèrent jusqu'à un numerus clausus afin de conserver leurs élèves et les retombées financière du suivi des stages pour leurs enseignants ...

Pour les enjeux généraux de la formation, il fallait tenir sur une approche commune à l'ensemble de l'école des SHS. Il est probable que la crainte diffuse de l'évaluation en préparation de la CTI joua, avec les grilles de compétences et un rôle attendu plus important et plus ambitieux des SHS. A cela s'ajoutait un besoin d'harmonisation et de simplification au sein de l'école, reconnu par beaucoup de collègues enseignants chercheurs de toutes disciplines, ainsi qu'une bonne compréhension des enjeux SHS par deux des cinq directeurs de département. Cela permit d'obtenir des résultats non négligeables. Une négociation d'ensemble au niveau de l'école relativement difficile institua le principe d'un « module matière HES » commun à tous les départements, c'est-à-dire un volume horaire unique et incontournable pour toute formation HES, avec une évaluation prise en compte dans le bilan annuel de chaque élève sous forme de crédits ECTS. Les enseignements auraient lieu soit dans le cadre d'un module HES complet de $21 \mathrm{~h}$, soit d'un demi-module de $10 \mathrm{~h} 30$. Ce point peut paraître mineur, mesquin même, mais en fait il organisait la rationalité de la grille horaire et en donnait sa pleine responsabilité à HES-IHT, qui en contrepartie devait mobiliser une secrétaire à temps quasi complet pour son fonctionnement pratique!

\subsection{La mise en place d'une maquette pédagogique}

Une autre négociation, porta sur le fait qu'il allait exister deux types de modules : ceux transversaux à l'école et ceux propres aux départements, en fonction de leurs besoins spécifiques. Ce point fut la pierre d'achoppement de nombreuses discussions. Il reposait sur le présupposé intellectuel que le département est seul capable de définir ses besoins, y compris dans des domaines de compétences comme les SHS ; mais aussi sur une réalité assez fréquente : le département disposait déjà de vacataires appréciés car pleinement conformes à son orientation pédagogique générale. Dans cette logique, il était essentiel pour lui de conserver en propre ces personnels, sans les perdre dans le « magma » HES de l'école... L'une des astuces, mais il en faut dans de telles négociations, fut de vendre sous le label d'une «base d'organisation département » des modules généraux communs et dont les contenus comme les enseignants, validés en Conseil des études de l'école, échappaient de fait au contrôle des départements. Le « tout compris » et le « sur mesure » annoncé du projet l'étaient bien, mais dans un cadre conceptuel défini par les collègues de HES-IHT. Cela permit, pour une part non négligeable, des enseignements SHS d'initiation et de culture générale pour l'ingénieur. Les autres étaient soit des options nouvelles proposées par HES-IHT, soit la continuation d'une activité département ancienne, mais intégrée dans le nouveau service. Il est clair que le soutien d'une direction d'école dans de telles situations est important; il est également clair que nous n'avons pas toujours bénéficié de ce soutien et que cela se traduisit par de nombreux combats d'arrière-garde... Une grille d'enseignement négociée patiemment au niveau du Conseil des études et dans chaque département voyait le jour en 2006, applicable à la rentrée 2007 pour les premières années ingénieurs (Tableaux 2 et 3), en 2008 pour les secondes années (Tableau 4) et en 2009 pour les troisièmes années (Tableau 5).

Le point fort du premier semestre à l'école fut le module1.1 de « Méthodologie et culture générale pour l'ingénieur », où chaque intervenant prenait les exemples dans son champ disciplinaire, mais dans un cadre méthodologique commun de travail sur documents et de pratique de la synthèse. C'était en

\footnotetext{
${ }^{9}$ La numérotation est apparemment irrégulière, parce que certains modules ont été déplacés ou supprimés. Comme la négociation impliquait un grand nombre de personnes, il a été décidé de maintenir les numérotations initiales pour éviter des confusions.
} 
Les sciences humaines dans les parcours scientifiques et techniques professionnalisants

Tableau 2. L'offre école du $1^{\mathrm{er}}$ semestre d'élève ingénieur (dit S5), rentrée 2007 (le statut remplace le nom).

\begin{tabular}{|c|c|c|}
\hline $\begin{array}{l}\text { Intitulé provisoire, responsable, } \\
\text { volume }\end{array}$ & $\begin{array}{l}\text { Objectifs, contenus, } \\
\text { compétences }\end{array}$ & Mise en œuvre, évaluation \\
\hline $\begin{array}{l}\text { HES 1.1 Méthodologie et culture } \\
\text { générale pour l'ingénieur } \\
2 \mathrm{M} \text { conf + prof + vacataires } \\
21 \mathrm{~h} \text { TD } \\
\text { UV école, gestion HES }\end{array}$ & $\begin{array}{l}\text { UV de méthodologie et de culture } \\
\text { générale } \\
\text { Recherche documents, analyse } \\
\text { synthèse } \\
\text { Expression écrite et orale. } \\
\text { Aborder une dimension } \\
\text { de l'entreprise dans le champ de } \\
\text { compétence de l'intervenant. }\end{array}$ & $\begin{array}{l}\text { Base département } \\
\text { Organisation en TD } 24 \text { élèves } \\
\text { Dossier écrit + soutenance } \\
\text { Séances prévues à la BU } \\
\text { Note individuelle }\end{array}$ \\
\hline $\begin{array}{l}\text { HES 1.39 Initiation au droit, droit } \\
\text { du travail } \\
\text { ATER droit demandé (ou M conf) } \\
1 / 2 \text { module: } 10 \text { h30 de cours } \\
\text { UV école, gestion HES }\end{array}$ & $\begin{array}{l}\text { Notions générales sur le droit, } \\
\text { vocabulaire. } \\
\text { Concepts de base du droit de } \\
\text { l'entreprise, le droit du travail } \\
\text { Préparation du stage ouvrier sur le } \\
\text { plan des règlements }\end{array}$ & $\begin{array}{l}\text { Base département } \\
\text { A mettre en place au niveau } \\
\text { département, } \\
\text { peut éventuellement se faire au } \\
\text { second semestre. } \\
\text { Note individuelle. }\end{array}$ \\
\hline $\begin{array}{l}\text { HES 1.5 Fondements de l'économie } \\
\text { et de la gestion } \\
\text { PRAG } 21 \mathrm{~h} \text { (TD ou cours, } \\
\text { niveau département) } \\
\text { UV école, gestion HES }\end{array}$ & $\begin{array}{l}\text { Fondements de l'économie, } \\
\text { économie générale et économie } \\
\text { de l'entreprise } \\
\text { Fondements de la gestion }\end{array}$ & $\begin{array}{l}\text { Base département. } \\
\text { Note individuelle }\end{array}$ \\
\hline $\begin{array}{l}\text { EPS } \\
\text { PRAG } \\
\text { Module } 21 \mathrm{~h} \\
\text { UV école, gestion HES }\end{array}$ & $\begin{array}{l}\text { Pratique sportive } \\
\text { Eléments sociologiques et } \\
\text { psychologiques du sport } \\
\text { Outil de formation humaine pour } \\
\text { l'ingénieur }\end{array}$ & $\begin{array}{l}\text { Base département } \\
\text { Note individuelle }\end{array}$ \\
\hline \multicolumn{3}{|c|}{ Total élève proposé : 73 h 1/2 } \\
\hline
\end{tabular}

réalité un effort très important à l'échelle des ressources humaines de HES, comme en termes financier pour l'école, afin de le réaliser en groupes de TD généralisés à tous les départements (12 groupes de TD à la rentrée 2007). Cette formule permettait d'adapter le projet à différents types d'intervenants et de fédérer la nouvelle équipe. A côté, un diptyque ou triptyque assez classique était proposé, en initiation à la gestion et à l'économie, au droit et l'incontournable et nécessaire pratique sportive.

Un effort particulier était fait en direction du second semestre de première année, en heures élèves et en spécificités HES. Une réflexion importante et constructive a eu lieu avec les départements, une fois admis les principes généraux déjà évoqués. Deux activités réellement transversales à l'école apparaissent : HES 1.7, avec deux options, une pour un rattrapage en français écrit ou oral pour les élèves détectés en difficulté au premier semestre via HES 1.1, ou à leur propre demande (étudiants étrangers), et l'autre proposant des cours électifs de SHS, c'est-à-dire des enseignements librement choisis par l'élève. Il s'agissait d'un véritable porte-drapeau de ce que notre équipe pouvait proposer et d'un appel au libre choix de l'élève qui s'exprimait dans un cadre autre que le département. Une véritable révolution culturelle à Polytech ${ }^{10}$ !

La création d'une journée HES commune à toute l'école, en fin d'année, formait le second volet de cet effort de transversalité. La première édition fut assez prometteuse, d'abord parce que les élèves s'y sont généralement bien impliqués. Ils devaient présenter leur projet de promotion (première partie du module HES 1.2) à toute l'école, sous forme d'expo d'affiches et de communication collective en amphi ; ils devaient également trouver un conférencier lors de leurs rencontres avec des professionnels. Ensuite, notamment pour une école à plusieurs sites (Nantes et Saint-Nazaire), les occasions de mixer

${ }^{10}$ Comment ne pas penser à Bourdieu et à son affirmation : « la sociologie est un sport de combat »! 
Tableau 3. L'offre du second semestre à l'école (S6), rentrée 2007.

\begin{tabular}{|c|c|c|}
\hline $\begin{array}{l}\text { Intitulé provisoire, responsable, } \\
\text { volume }\end{array}$ & $\begin{array}{l}\text { Objectifs, contenus, } \\
\text { compétences }\end{array}$ & Mise en œuvre, évaluation \\
\hline $\begin{array}{l}\text { HES } 1.2 \text { Découverte de } \\
\text { l'entreprise, projet de promo } \\
\text { Responsable : EC ou ATER } \\
\text { Coordination dép. : responsable } \\
\text { HES + représentant département. } \\
15 \mathrm{~h} \text { TD ou cours }+6 \mathrm{~h} \text { « journée des } \\
1 \text { ères années » }=21 \mathrm{~h} \\
\text { UE école, gestion HES }\end{array}$ & $\begin{array}{l}\text { Découverte des entreprises et des } \\
\text { marchés de la spécialité. } \\
\text { Visites d'entreprises, rencontres de } \\
\text { professionnels. } \\
\text { CV et marché de l'emploi } \\
\text { Conférences pro. lors de la Journée } \\
\text { des 1ères années. }\end{array}$ & $\begin{array}{l}\text { Projet défini au niveau du } \\
\text { département. } \\
=\sum \text { mini projets par binôme ou } \\
\text { trinôme } \\
\text { Présentation finale du projet } \\
\text { dép. = Journée des 1ères années } \\
\text { Besoin d'une journée commune } \\
\text { école } \\
\text { Note individuelle sur le projet }\end{array}$ \\
\hline $\begin{array}{l}\text { HES 1.6 Technique et méthodes } \\
\text { des SHS pour l'ingénieur } \\
\text { L'enseignant est responsable de son } \\
\text { option } \\
21 \mathrm{~h} \text { (TD ou cours) ou } 1 / 2 \text { module } \\
\text { gestion HES ou département }\end{array}$ & $\begin{array}{l}\text { A- Risque et qualité (PAST) } \\
\text { B- Comptabilité (PRAG) } \\
\text { C- Communication au sein } \\
\text { de l'entreprise - insertion } \\
\text { professionnelle (PAST) } \\
\text { D- Connaissance des métiers et } \\
\text { de l'entreprise (responsable à } \\
\text { déterminer) }\end{array}$ & $\begin{array}{l}\text { en fonction des besoins } \\
\text { spécifiques des départements } \\
\text { Un thème ou deux thèmes peuvent } \\
\text { être retenus, ils forment dans ce cas } \\
\text { chacun un demi module HES } \\
\text { Note individuelle }\end{array}$ \\
\hline $\begin{array}{l}\text { HES 1.7 Cours électifs du 2d } \\
\text { semestre, culture générale de } \\
\text { l'ingénieur } \\
\text { Responsable : Prof ou M. Conf } \\
\text { - Option A : sur liste suite à HES } \\
1.1 \\
\text { - Option B : au choix de l'élève } \\
21 \text { h de cours } \\
\text { UE école, gestion HES } \\
\text { - Option C : choix d'un cours } \\
\text { unique par le département } \\
21 \text { h de cours } \\
\text { UE gestion département }\end{array}$ & $\begin{array}{l}\text { Option A : Renforcement de } \\
\text { l'expression écrite et orale pour les } \\
\text { élèves repérés en difficultés suite à } \\
\text { HES 1.1 } \\
\text { Option B : cours électifs } \\
\text { a- Marché du travail, qualification } \\
\text { et compétence (M Conf) } \\
\text { b- Histoire des techniques } \\
\text { (M conf) } \\
\text { c- Géopolitique et mondialisation } \\
\text { (vacataire) } \\
\text { d- Environnement et } \\
\text { développement durable (prof) } \\
\text { e- Design et industrie (vacataire } \\
\text { Ecole de design) } \\
\text { Option C : enseignement organisé } \\
\text { sur la base du département }\end{array}$ & $\begin{array}{l}\text { A et B : Mise en place au niveau } \\
\text { école, } \\
\text { Site de Nantes, plage transversale } \\
\text { d' } 1 \text { h } 1 / 2 \\
\text { Principe du libre choix de l'élève } \\
\text { (sauf choix contraire du } \\
\text { département) }\end{array}$ \\
\hline EPS, PRAG, module $21 \mathrm{~h}$ & Pratique sportive & Note individuelle \\
\hline \multicolumn{3}{|c|}{ Total élève proposé : 84 h } \\
\hline
\end{tabular}

les élèves dans une activité commune est peu fréquente, d'où normalement un réel soutien de la direction à ce type d'initiative. On peut bien entendu remarquer qu'il s'agit d'abord d'une animation collective plus que d'une formation, mais ce n'est finalement pas si loin d'un colloque ou d'un salon et cela se marie fort bien à la culture d'une école d'ingénieur. Le travail préparatoire de découverte d'un secteur professionnel, de son marché économique voire de sa géopolitique a également passionné nombre d'élèves. Il faut aussi signaler la transversalité de la pratique sportive qui, bloquant une plage d'une après-midi, était finalement la seule activité transversale à l'école depuis son origine, entre les quatre départements nantais.

L'idée générale de la seconde année ingénieur est d'une part de consolider les besoins classiques à caractère opératoire exprimés par les départements, mais de continuer d'autre part à offrir un choix notable de formations à caractère plus ouvert, donnant accès à cette culture générale de l'ingénieur qui nous semblait essentielle. Bien entendu, il s'agit d'un menu et la troisième partie de la négociation, après les principes généraux de l'intervention puis l'organisation de l'offre, fut les choix des départements ... Nous y reviendrons dans suite de l'article. 
Les sciences humaines dans les parcours scientifiques et techniques professionnalisants

Tableau 4. Semestres 7-8 (2ème année d'élèves ingénieurs), rentrée 2008.

\begin{tabular}{|c|c|c|}
\hline $\begin{array}{l}\text { Intitulé provisoire, responsable, } \\
\text { volume }\end{array}$ & $\begin{array}{l}\text { Objectifs, contenus, } \\
\text { compétences }\end{array}$ & Mise en œuvre, évaluation \\
\hline $\begin{array}{l}\text { HES 2.1 Jeux d'entreprise } \\
\text { PRAG } \\
\text { Module : } 21 \mathrm{~h} \text { TD sur } 3 \text { jours }\end{array}$ & $\begin{array}{l}\text { Compétences : Sensibilisation à la } \\
\text { gestion de l'entreprise et à ses } \\
\text { différents champs } \\
\text { Techniques et con conceptuels }\end{array}$ & $\begin{array}{l}\text { Base département } \\
\text { Note individuelle } \\
\text { UE école, gestion HES }\end{array}$ \\
\hline $\begin{array}{l}\text { HES 2.2 Debreifing du jeu } \\
\text { d'entreprise } \\
\text { PRAG ou vacataire ou PAST } \\
1 / 2 \text { module }: 10 \mathrm{~h} 30\end{array}$ & $\begin{array}{l}\text { Une série de conférences par des } \\
\text { pro. : gestion financière, ressources } \\
\text { humaines, marketing, choix } \\
\text { technologiques ... }\end{array}$ & $\begin{array}{l}\text { Base département } \\
\text { Note individuelle } \\
\text { UE école, gestion HES }\end{array}$ \\
\hline $\begin{array}{l}\text { HES 2.3 Gestion de projet } \\
\text { Prof }(+ \text { vacataires départements) } \\
\text { Module de } 21 \mathrm{~h} \text { ou } 1 / 2 \text { module de } \\
10 \mathrm{~h} 30\end{array}$ & $\begin{array}{l}\text { a- préparation au projet } \\
\text { b- implication des SHS dans les } \\
\text { projets dep. } \\
\text { c- suivi de stages, part des SHS }\end{array}$ & $\begin{array}{l}\text { Base département } \\
\text { Note individuelle }\end{array}$ \\
\hline $\begin{array}{l}\text { HES 2.4 Culture internationale } \\
\text { PRAG, } 1 / 2 \text { module }\end{array}$ & $\begin{array}{l}\text { Initiation à l'inter-culturalité } \\
\text { (PRAG + collègue de langues) }\end{array}$ & $\begin{array}{l}\text { Base département } \\
\text { Note individuelle }\end{array}$ \\
\hline $\begin{array}{l}\text { HES } 2.5 \text { Techniques et méthodes } \\
\text { des SHS, orientation gestion et } \\
\text { marketing } \\
\text { (suite HES } 1.6 \text {, même organisation) } \\
\text { PRAG Module de } 21 \mathrm{~h} \text { ou } \\
1 \text { module de } 10 \mathrm{~h} 30\end{array}$ & $\begin{array}{l}\text { a- comptabilité (PRAG) } \\
\text { b- mercatique (PRAG) } \\
\text { c- gestion de production }+ \\
\text { dimension du département } \\
\text { (vacataire choix du département) }\end{array}$ & $\begin{array}{l}\text { Choix par les départements } \\
\text { Note individuelle }\end{array}$ \\
\hline $\begin{array}{l}\text { HES 2.6 Cours électifs, culture } \\
\text { générale de l'ingénieur } \\
\text { (Suite de HES } 1.7 \text {, même } \\
\text { organisation) Prof ou ATER } \\
1 \frac{2}{2} \text { module de } 10 \mathrm{~h} 30,6 \text { conf. et } \\
1 \text { évaluation }\end{array}$ & $\begin{array}{l}\text { a- éthique (M conf) } \\
\text { b- développement durable } \\
\text { (niveau 2) (prof) } \\
\text { c- histoire des ingénieurs (ATER) } \\
\text { d- sociologie de l'innovation } \\
\text { (M conf ou vacataire) } \\
\text { e- aménagement du territoire } \\
\text { industriel (M conf) } \\
\text { f- Projet de création d'entreprise } \\
\text { (vacataire et relations industrielles) }\end{array}$ & $\begin{array}{l}\text { Base école } \\
\text { Au choix des élèves } \\
\text { Note individuelle } \\
\text { UE école, gestion HES }\end{array}$ \\
\hline $\begin{array}{l}\text { HES } 2.7 \text { Techniques et méthodes } \\
\text { des SHS, droit et normes de } \\
\text { l'industrie } \\
\text { Responsable à déterminer par } \\
\text { département Module de } 21 \mathrm{~h} \\
\text { ou } 1 / 2 \text { module de } 10 \mathrm{~h} 30\end{array}$ & $\begin{array}{l}\text { a- qualité et propriété industrielle } \\
\text { (Prof et relations industrielles) } \\
\text { b- droit des contrats, brevets } \\
\text { (M conf) }\end{array}$ & $\begin{array}{l}\text { Base département } \\
\text { Note individuelle }\end{array}$ \\
\hline $\begin{array}{l}\text { HES } 2.8 \text { Techniques et méthodes } \\
\text { des SHS, orientation R \& D } \\
\text { Responsable à déterminer par } \\
\text { département Module de } 21 \mathrm{~h} \\
\text { ou } 1 / 2 \text { module de } 10 \mathrm{~h} 30\end{array}$ & $\begin{array}{l}\text { a- intelligence économique } \\
\text { (PRAG) } \\
\text { b- industrialisation (PRAG) }\end{array}$ & $\begin{array}{l}\text { Base département } \\
\text { Note individuelle }\end{array}$ \\
\hline $\begin{array}{l}\text { HES 2.9 Communication et } \\
\text { entreprise } \\
\text { M conf }+ \text { vacataire dép. } \\
\text { Module de } 21 \mathrm{~h} \text { ou } 1 \frac{1}{2} \text { module de } \\
10 \mathrm{~h} 30\end{array}$ & à préciser par l'enseignant & $\begin{array}{l}\text { Base département } \\
\text { Note individuelle }\end{array}$ \\
\hline EPS, PRAG, module $21 \mathrm{~h}$ & Pratique sportive & Note individuelle \\
\hline
\end{tabular}

La pratique transversale école, avec l'avantage de mixer les élèves, restait ensuite, en seconde année ingénieurs comme en troisième, cantonnée à un module de cours optionnels qui reprenait les mêmes principes qu'en première année. La difficulté était là de trouver une plage horaire commune aux quatre départements du site de Nantes, c'est-à-dire de l'imposer en préalable à la rédaction de chaque emploi 
Tableau 5. Semestres 9-10 (5 $5^{\text {èmes }}$ années), en jaune : le stage de fin d'études sous la responsabilité HES-IHT.

\begin{tabular}{|c|c|c|}
\hline $\begin{array}{l}\text { Intitulé provisoire, responsable, } \\
\text { volume }\end{array}$ & $\begin{array}{l}\text { Objectifs, contenus, } \\
\text { compétences }\end{array}$ & Mise en œuvre, évaluation \\
\hline $\begin{array}{l}\text { HES 3.1 Conférences et séminaires } \\
\text { A : Base école : } 1 / 2 \text { module } \\
\text { B : Saint-Nazaire, enseignement } \\
\text { spécifique : } 1,5 \text { Module, } 31,5 \mathrm{~h} \text { TD }\end{array}$ & $\begin{array}{l}\text { Culture générale métier } \\
\text { Sensibilisation à des questions } \\
\text { professionnelles } \\
\text { Sensibilisation à des questions } \\
\text { culturelles et sociétales }\end{array}$ & $\begin{array}{l}\text { Plage transversale école } \\
\text { Rédaction d'une note de synthèse } \\
\text { en temps limité, note individuelle }\end{array}$ \\
\hline $\begin{array}{l}\text { HES 3.2 Gestion des ressources } \\
\text { humaines } \\
\text { M. conf } \\
\text { Module ou } 1 / 2 \text { module }\end{array}$ & $\begin{array}{l}\text { a- Conflits et négociations } \\
\text { b- Ressources humaines } \\
\text { c- Conduite de réunion } \\
\text { d- Management d'équipe } \\
\text { e- Management }\end{array}$ & $\begin{array}{l}\text { Base département } \\
\text { Note individuelle }\end{array}$ \\
\hline $\begin{array}{l}\text { HES 3.3 Outils financiers } \\
\text { PRAG } \\
\text { Module ou } 1 / 2 \text { module }\end{array}$ & $\begin{array}{l}\text { Familiarisation avec les outils } \\
\text { financiers de l'entreprise } \\
\text { Initiation }\end{array}$ & $\begin{array}{l}\text { Base département } \\
\text { Note individuelle }\end{array}$ \\
\hline $\begin{array}{l}\text { HES 3.4 Insertion professionnelle } \\
\text { M. Conf et ATER } \\
\text { Module ou } 1 / 2 \text { module }\end{array}$ & $\begin{array}{l}\text { a- Insertion professionnelle } \\
\text { b- Simulation d'embauche } \\
\text { (horaires groupés) } \\
\text { c- Droit, insertion professionnelle } \\
\text { et management } \\
\text { d- projet création d'entreprise }\end{array}$ & $\begin{array}{l}\text { Base département } \\
\text { Note individuelle }\end{array}$ \\
\hline $\begin{array}{l}\text { HES 3.5 Marketing, PRAG } \\
1 / 2 \text { module }\end{array}$ & Initiation & $\begin{array}{l}\text { Base département } \\
\text { Note individuelle }\end{array}$ \\
\hline $\begin{array}{l}\text { HES 3.6 Retour d'expérience, stage } \\
\text { à l'étranger } \\
\text { Responsabilité partagée avec les } \\
\text { Langues }\end{array}$ & $\begin{array}{l}\text { Capitalisation des connaissances } \\
\text { Dimension interculturelle du } \\
\text { stage }\end{array}$ & $\begin{array}{l}\text { Base département } \\
\text { Rapport spécifique et moment de } \\
\text { la soutenance stage. } \\
\text { Note individuelle }(\mathrm{en}+\text { stage })\end{array}$ \\
\hline $\begin{array}{l}\text { HES 3.7 Dimension SHS du projet } \\
\text { de fin d'études } \\
\text { Coordinateur département? }\end{array}$ & $\begin{array}{l}\text { Place des SHS dans le projet de } \\
\text { fin d'études }\end{array}$ & $\begin{array}{l}\text { Base département } \\
\text { Partie du rapport et moment de la } \\
\text { soutenance. Note fin de stage. }\end{array}$ \\
\hline $\begin{array}{l}\text { HES } 3.8 \text { Projet de fin d'étude dans } \\
\text { le cadre de l'Option transversale } \\
\text { MORI } \\
\text { Prof }\end{array}$ & $\begin{array}{l}\text { Management des organisations et } \\
\text { des risques industriels. }\end{array}$ & $\begin{array}{l}\text { Base IHT } \\
\text { Note fin de stage }\end{array}$ \\
\hline EPS, PRAG & Pratique sportive & Note individuelle \\
\hline
\end{tabular}

du temps par chaque département. Il faut noter qu'en troisième année, ce module dit «transversal école » devait prendre la forme de conférences au cours du premier semestre, dont l'organisation était encore à prévoir au moment de la rédaction de ce projet. Sinon, l'offre de troisième année concrétisait un triple effort : le projet de fin d'étude transversal école MORI déjà évoqué et mis en place dès 2006, était confirmé ; une recherche d'adéquation entre les propositions HES et les demandes des départements par l'ouverture prévue de nombreuses options possibles au sein des modules; enfin une réflexion sur la place des SHS et de l'équipe HES au sein du rapport de stage de fin d'étude. Pour ce dernier point, nous n'étions pas du tout certains de disposer de suffisamment de personnels pour réellement faire face à la situation, ni de disposer des crédits horaires nécessaires pour y parvenir. C'était surtout une affirmation de principe au sein de l'école, sur la continuité du besoin des SHS à chaque étape de la formation des élèves. Par contre, envisager une dimension interculturelle spécifique pour les stages à l'étranger, en commun avec le service des Langues paraissait à l'époque un horizon plus réaliste.

\subsection{Les choix des départements}

Le cadre que nous venons d'évoquer formait un «menu à la carte » pour les départements, et il était validé par le Conseil des études de l'école. Si l'on ajoute la pression des horaires recommandés par la 
CTI, les départements devaient à leur tour faire des choix relativement contraints ! La négociation a porté d'un coup sur les trois années, pour pouvoir proposer des ensembles cohérents aux étudiants qui entraient dans le système, même si sa mise en place réelle devait s'étaler sur trois ans avec d'inévitables ajustements qui furent cependant limités ${ }^{11}$. Il s'agissait également de satisfaire à l'évaluation de la CTI afin de lui présenter une perspective globale des contenus des formations proposées pour les évaluer.

Pour faciliter le fonctionnement pratique, très vite un responsable HES par département a été envisagé. Il s'agissait autant d'une modalité pratique pour l'organisation des cours que d'une revendication des départements afin d'avoir un interlocuteur privilégié. Ce fut parfois une forme de retour en arrière, certains départements cherchant à phagocyter le responsable HES et à se l'approprier en tant qu'organisateur local, en court-circuitant l'échelon école que commençait à véritablement représenter le service HES dans son ensemble.

Donnons quelques lignes de force de ces choix. En première année, HES 1.1, module d'introduction aux SHS en école d'ingénieur et de méthodologie générale, a joué le rôle d'un TD d'initiation obligatoire. Pierre angulaire de l'édifice, il fut finalement choisi par tous les départements ; il mobilisa un effort considérable de l'équipe HES, et il fut assez bien accepté par les élèves. Un élément de pression pour ce choix fut d'expliquer qu'un tel module servait à préparer à la rédaction des rapports, et à détecter en amont les élèves en difficulté en français écrit et oral, enfin à leur proposer une solution de mise à niveau au second semestre, dans le cadre des options transversales (HES 1.7, option A). Dans une école comme Polytech, la diversité des recrutements explique que certains élèves soient en retard en français et qu'ils arrivent par des filières scientifiques ou technologiques à un honnête bac plus trois sans avoir comblé cette lacune. Les élèves étrangers sont aussi concernés et un cours de français langue étrangère a été organisé dans ce cadre, avec le soutien du département de la faculté des Lettres spécialisé dans ce type de formations. Quatre groupes d'une douzaine d'élèves ont fonctionné dans le cadre HES 1.7A, la première année, avec français écrit, français langue étrangère, et deux groupes d'expression orale animés par des comédiens professionnels, soit un gros quart des effectifs de la promotion!

Le couplage escompté avec l'autre partie de HES 1.7B, les cours électifs, n'a cependant pas fonctionné aussi bien qu'escompté pour la première année d'expérimentation. Pourtant, j'ai souvent eu l'occasion de constater l'efficacité de ce type d'enseignement en école, qui en appelant au libre choix des élèves évite l'écueil de l'obligation et engendre une attitude généralement plus constructive et plus ouverte. Dans ce sens, il faut signaler l'intérêt pour « design et industrie », alors que l'école offrait des formations de spécialités techniques pures et dures, et qu'a priori rien ne poussait les élèves dans cette direction... Un seul département a spontanément choisi cette formule sur les quatre possibles, la transversalité ne pouvant être organisée avec Saint-Nazaire. Ce fut, pour notre équipe, l'un des demiéchecs de la mise en place de la réforme. Le point positif était qu'un des départements acceptait de jouer le jeu, malgré la contrainte horaire refusée par les autres, permettant une expérimentation que l'on prévoyait fructueuse au vu de son existence dans d'autres écoles.

En termes horaires, tous les départements choisirent pour leurs premières années la pratique sportive sur deux semestres, soit deux modules HES, confirmant son caractère historique et traditionnel au sein d'une école comme Polytech Nantes, au moins en première année, avec un rôle intégrateur évident des élèves entre eux. En dehors, les totaux des modules HES choisis se sont situés à cinq sur l'année (3 départements) ou six (2 départements), ce qui correspond à une obligation horaire de 18 à $21 \%$. En tenant compte des langues autour de 8-10\%, on arrivait aux $30 \%$ recommandés par la CTI pour deux des départements, ceux qui avaient le mieux joué le jeu de la réforme, à un peu moins pour les autres.

Les choix de seconde année maintenaient un bon niveau de modules HES sur l'année, avec une part importante de cours adaptés aux besoins spécifiques des départements. Les taux restent à 5 ou 5,5 enseignements par année, mais ils baisent en pourcentage horaires par le jeu des demi-modules. La

\footnotetext{
${ }^{11}$ En tant que responsable de la création de la maquette, je n'ai préparé que deux des trois rentrées concernées, la dernière le fut par mon successeur.
} 
variable d'ajustement horaire des départements tend à être la pratique sportive ; celle-ci n'était plus que pendant un semestre et même carrément abandonnée par l'un des départements. Parallèlement, les cours de spécialités scientifiques et technologiques tendent à augmenter assez sérieusement en seconde année, jugé comme le meilleur moment pour cela, au-delà des obligations de la CTI. Les pourcentages des interventions HES tout compris tombent entre 12,5 et $17 \%$. Si l'on tient compte d'un tassement similaire des Langues d'un ou deux pour cent, nous voyons que l'on décroche cette fois assez nettement de recommandation CTI, au mieux 25-26\% au pire à peine $20 \%$ !

Dans le domaine des satisfactions, tout d'abord l'équipe réunie entre l'ancien HES et l'IHT permit de faire face en interne à la plupart des besoins exprimés, pour le moins de coordonner efficacement les vacataires et d'assainir plusieurs situations ponctuelles d'anciens cours quasiment incontrôlés. Ensuite, trois départements nantais sur quatre choisissent les cours électifs en seconde année, il est vrai sur un demi module, ce qui est assez léger, mais c'est une avancée incontestable. Le module complet de $1^{\text {re }}$ année était jugé trop lourd et trop précoce. En ce sens, des SHS mieux identifiées apparaissent à leur tour, dans une école d'ingénieurs, comme des spécialités optionnelles, ce qui n'est pas insensé. Le troisième point positif est l'entrée des SHS dans les projets des élèves de seconde année, parfois à la demande des départements. Enfin, il reste une belle marge de progression pour les années à venir afin de rejoindre un jour l'horizon des $30 \%$, à la condition que l'école parvienne à rogner les débordements horaires des niches de spécialistes... toutes plus indispensables les unes que les autres dans une école de spécialistes !

La dernière année est bien entendue marquée par le stage de fin d'études, au second semestre, où le principe de la participation de HES à l'évaluation des stages est acté. Seul le premier semestre forme donc une base pour les choix et une évaluation de l'impact horaire des SHS. Certes l'échéance était encore lointaine au moment de ces engagements de principe, mais les indications sont nettes et elles montrent une vraie divergence entre départements. La pratique sportive a presque disparue, un seul département la conservant dans son planning, un autre hésitait. Seulement deux départements restent sur une perspective pleinement compatible avec les remarques de la CTI, ceux qui ont vraiment joué le jeu, dès la première année, les trois autres décrochent nettement.

\section{Esquisse d'un contrepoint : Les « humanités " à l'U.T.B.M.}

\subsection{Le département des Humanité de I'UT Belfort-Montbéliard au début des années 2000}

Chronologiquement parlant, mon expérience des SHS à Belfort-Montbéliard, comme directeur du département des Humanités, est antérieure à celle de Polytech Nantes, ce que j'ai parfois vécu comme une régression en tant que directeur, mais aussi comme possédant un excellent point de référence pour instiller une réforme. Disons tout de suite que le format spécifique de ces enseignements a été prévu dès l'origine de la première université de technologie (UT) à Compiègne (Lamard et Lequin 2006) ${ }^{12}$, d'une manière parfaitement transversale à l'école, et qu'il a été solidement organisé à Belfort, sur ce modèle, dans le cadre initial de l'IPSE de Sévenans, par Gilbert Karpman et Pierre Lamard ${ }^{13}$. En écho aux meilleures expériences mondiales, notamment au MIT, les fondateurs de l'UTC font de l'enseignement des SHS en école d'ingénieur l'une des priorités de leur projet, dont le résultat, trente ans après, est un modèle abouti largement en avance sur celui que nous venons de décrire. Il s'agit très certainement d'un exemple remarquable et peut-être même exceptionnel dans le paysage français des écoles d'ingénieur au tournant des années 2000. Cet apogée fut sanctionné par la création du laboratoire RECITS (Recherches

\footnotetext{
12 Voir également dans ce volume, Charles Lenay et collaborateurs, Pour une recherche technologique en Sciences Humaines et Sociales.

${ }^{13}$ Respectivement premier directeur de l'IPSE (antenne de l'UTC sur le Territoire de Belfort) et premier responsable du département des Humanités. L'IPSE devient l'UTBM suite à l'absorption de l'ENI Belfort (rentrée 1999).
} 
Les sciences humaines dans les parcours scientifiques et techniques professionnalisants

Tableau 6. Les UV existantes au département des Humanités de l'UTBM (rentrée 2001).

\begin{tabular}{|l|c|l|c|}
\hline \multicolumn{2}{|c|}{ Culture générale : 60 U.V. } & \multicolumn{2}{c|}{ Langues \& communication : 36 U.V } \\
\hline Economie \& environnement & 3 & Anglais & 9 \\
\hline Gestion & 5 & Allemand & 6 \\
\hline Droit & 4 & Espagnol & 6 \\
\hline Sociologie & 6 & Russe & 2 \\
\hline Philosophie & 4 & Italien & 2 \\
\hline Europe et relations internationales & 6 & Chinois & 2 \\
\hline Communication & 7 & Coréen & 2 \\
\hline Histoire et histoire des techniques & 11 & Langue validée par une université étrangère & 1 \\
\hline Arts \& design & 5 & Expression \& Communication en français & 6 \\
\hline Sport & 6 & & \\
\hline Recherche et travaux personnes & 3 & & \\
\hline
\end{tabular}

et études sur les choix industriels, technologiques et scientifiques), immédiatement reconnu comme « Jeune équipe » de recherches par le Ministère de l'enseignement supérieur.

Le système d'organisation est radicalement différent, en premier lieu par un service pédagogique central commun à tous les départements et en charge de l'ensemble des questions d'emploi du temps. En second lieu, il est différent au niveau des enseignements de SHS, ici appelés "Humanités », organisés en département autonome commun avec les langues. Cette notion d'autonomie vaut tant pour l'offre d'enseignements, validée par le seul Conseil des études de l'UT, que pour l'étudiant qui choisit individuellement et librement ses enseignements, indépendamment de son rattachement à un département «technique ». L'offre est fortement diversifiée, ce qui ne veut pas dire qu'elle est parfaitement équilibrée, avec par exemple un effet de distorsion en faveur des historiens lié à la création de RECITS à Belfort. C'est la touche spécifique de l'UTBM dans le domaine des SHS, ici tournées vers l'histoire technique et industrielle, l'histoire économique et de l'aménagement des territoires industriel, vers le patrimoine industriel également.

Les enseignements sont organisés par unité de valeur (UV) de 24 h, ouvrant droit de manière égale aux crédits ECTS, après un contrôle des connaissances réussi, sous la responsabilité de l'enseignant en charge de l'UV. L'étudiant est invité à choisir une moyenne de trois UV par semestre de présence à l'école, au sein d'un catalogue publié annuellement et plutôt bien pourvu (Tableau 6). Il s'agit d'une liberté guidée par des pré-requis en termes de suite d'UV au sein d'une discipline de SHS, ainsi que par le numérus clausus de la plupart des UV, généralement formatées sur le principe d'un groupe de TD à 24 élèves. Une possibilité de projet libre existe également, accompagné par un tuteur et validé par un jury. L'étudiant choisit son parcours personnel au fur et à mesure de sa formation au sein de l'UT, y compris pendant le premier cycle préparatoire où les UV de culture générale et de langues forment un ensemble autonome de sa formation, mais indispensable à sa validation finale. Les possibilités de choix pour l'étudiant dépendent de l'offre en UV et des possibilités d'y accéder, et de rien d'autre. Elles ne dépendent pas de son niveau de parcours dans le premier cycle ou dans une formation d'ingénieur. L'étudiant suit les seules recommandations du département des Humanités quant aux pré-requis de certaines unités de valeur et pour des possibilités de parcours renforcés aboutissant à la possibilité de «mineurs » de formation. Un mineur est validé par le département et il est porté sur le diplôme d'ingénieur UTBM.

Pour être complet, cette liberté très large au sein d'un choix assez vaste a été critiquée par la CTI, lors de ses évaluations, qui avaient recommandé de rendre obligatoire certains enseignements de base, notamment en économie-gestion. Les Langues avaient déjà une pratique d'obligation d'UV pour atteindre les niveaux requis, dont seuls les étudiants de bon niveau étaient dispensés au profit d'un choix plus vaste d'approfondissements. Il faut également signaler que la question des plages horaires dédiées aux SHS était réglée avec beaucoup d'expérience par le service central des études, mais qu'il y avait parfois des incompatibilités avec certains enseignements de branche, ce qui réduisait les possibilités 


\section{SHS Web of Conferences}

Tableau 7. Les ressources humaines du département des Humanités de l’UTBM.

\begin{tabular}{|l|c|}
\hline \multicolumn{2}{|l|}{ L'équipe du département des Humanités de l'UTBM (rentrée 2001) } \\
\hline Professeurs & 2 \\
\hline Maîtres de conférences & 9 \\
\hline Enseignants-chercheurs contractuels & 6 \\
\hline PRAG et certifiés de l'enseignement secondaire & 12 \\
\hline PAST & 3 \\
\hline Maîtres de langues (étrangers diplômés dans leur pays) & 6 \\
\hline ATER & 2 \\
\hline Vacataires et conférenciers & $\approx 30$ \\
\hline Secrétariat & 2 \\
\hline
\end{tabular}

effectives de choix, notamment en $2^{\text {ème }}$ et $3^{\text {ème }}$ années ingénieur. Dans le fonctionnement pratique, certaines unités de valeur très demandées ou fortement recommandées étaient répétées à plusieurs reprise, dans des créneaux horaires différents et aussi sur les deux sites de l'école à ce moment-là, Belfort et Sévenans, comme les enseignements fondamentaux d'économie et de gestion ou encore les UV d'anglais préparant au TOEIC.

\subsection{Des ressources humaines au service de « vraies " SHS ?}

Le tableau 6 de l'offre d'enseignements est à compléter par celui des ressources humaines du département du tableau 7, qui indiquent ensemble le niveau tout à fait remarquable et significatif des efforts de l'UTBM sur la longue durée, avec des créations de postes régulières, notamment de maitres de conférences dans les différents domaines des SHS.

Il s'agit donc d'une offre adossée à un personnel important d'enseignants-chercheurs qualifiés dans des champs précis des SHS académiques : histoire, histoire des sciences et des techniques, sociologie, économie, gestion, philosophie et épistémologie, droit, communication, anglais, etc. La diversité est élevée et le souci d'une réelle initiation à différents domaines des SHS, en lien avec les métiers de l'ingénieur et le monde de l'entreprise, est une réalité pédagogique. Schématiquement le département se présente alors comme offrant deux grands domaines complémentaires : la culture générale de l'ingénieur et l'expression - communication ; l'inter-culturalité formant un domaine partagé avec certaines spécialités « maison » comme les initiations aux langues et aux civilisations extrêmeorientales, ce qui à l'époque était pionnier en école d'ingénieur.

Le département présentait alors l'offre de culture générale comme répondant à une série de domaines de formations proposés aux étudiants, domaines se déclinant eux-mêmes en sous-thèmes correspondant à des unités de valeur. L'idée générale était de stimuler l'appétit de connaissance par l'offre, ainsi que la suggestion d'un parcours personnel à construire au long de sa formation, celle-ci pouvant aller jusqu'à cinq ans pour un élèves entré après le bac en cycle préparatoire de l'UT. Trois grands groupes de parcours personnels se dessinent. Le premier concerne les acquisitions de connaissances fondamentales dans de grands domaines (économie, droit, histoire, géopolitique...), avec des accents sur des points plus précis pouvant être en rapport avec des projets personnels (institutions européennes, brevets...). Le second est une offre d'initiation à des outils et des méthodes de base (sciences de gestion, comptabilité, commerce international...) ouvrant là encore vers des thématiques plus précises et des approfondissements personnels ( $3^{e}$ langue, inter-culturalité, sociologie du travail...). Le troisième se tourne vers la culture technique, l'histoire de l'entreprise et de l'innovation (histoire économique, histoire des techniques, sociologie, culture technique ${ }^{14} \ldots$ ) et par des approfondissements

\footnotetext{
14 Il s'agit d'un enseignement spécifique aux UT, créé par Yves Deforges à l'ouverture de l'UTC, et que nous avons repris et développé à l'UTBM, sous forme de réflexion critique sur les objets et leurs évolutions, à partir des méthodes de la génétique technique. Elle fut considérée par beaucoup d'étudiants de cette époque comme initiatique à l'esprit des UT.
} 
plus spécialisés (patrimoine technique et industriel, veille technologique et intelligence économique ...). Les Humanités offraient également des spécialités partagées avec des entités voisines (entreprise, département technique) pour des ouvertures souvent très appréciées comme l'initiation au design et à l'ergonomie, les maquettes numériques de patrimoine technique, etc., dans des domaines frontières entre SHS et SPI. Ces domaines générant par eux-mêmes de la recherche expérimentale et alimentant les laboratoires de l'école.

Il se dégage de ce tableau, en tout cas pour moi-même, le sentiment d'une complétude assez heureuse, en particulier comme enseignant d'histoire et d'épistémologie des techniques ainsi que comme directeur du département pendant quatre années consécutives, mais aussi comme chercheur au sein du laboratoire RECITS. La plupart des questions posées par les SHS en école d'ingénieurs, qui sont ailleurs de rudes combats et l'objet de compromis pas toujours réussis, sont ici résolues dans le cadre de la vie normale de l'établissement, parce qu'elles ont été institutionnalisées au départ et que le contexte généraliste revendiqué par l'école permet de les intégrer dans une structure transversale puissante. La déconnection d'avec les départements en termes de gestion des enseignements permet d'avoir un véritable projet des SHS en école, sur la durée, projet qui se démarque plus facilement d'un utilitarisme trop étroit comme des contraintes d'emploi du temps au sein d'écoles dominées par un fonctionnement départemental. L'étudiant apprécie sa liberté de choix, même si en pratique elle est un peu plus contrainte qu'annoncée et même s'il a parfois un peu de mal à la gérer à ses débuts. Cette indépendance structurelle des Humanités n'implique pas du tout d'ignorer les départements techniques, mais d'avoir avec eux et avec leurs laboratoires des échanges plus sains, plus normaux, car dégagés des conflits d'intérêts de notre premier exemple.

Nous avons vu toutefois que la CTI n'était pas sans critique par rapport à ce modèle, et qu'il pouvait toucher à certaines limites. Par exemple, où s'arrête la liberté de choix ?, et n'y a t'il pas quelques enseignements d'initiation à imposer pour tous à leur arrivée, comme cela se fait déjà pour les langues ? En pratique, il est aussi arrivé qu'une UV soit créée à la demande express d'un département, tout en rentrant dans le système général et en la rendant accessible à tous les autres étudiants. Le coût d'un tel modèle est également élevé, et il a incontestablement un caractère privilégié. L'absence de développement du réseau des UT, en panne depuis les années 2000 malgré de beaux projets, ne le doit pas qu'à l'hostilité viscérale des grandes universités classiques à son égard, mais aussi à cette question des coûts de fonctionnement. Un tel contexte a également conduit vers un modèle d' « université forteresse », comptant essentiellement sur ses forces internes et ayant un certain mal à la fois géographique et culturel à s'adjoindre des compétences extérieures de qualité.

$$
* * *
$$

Au cours de nos deux articles, et notamment dans les deux comptes rendus d'expériences personnelles de celui-ci, nous avons observé la difficulté à définir des contenus de sciences humaines et sociales rigoureux, épistémologiquement bien définis et en même temps bien adaptés aux élèves ingénieurs, un public spécifique en regard de ces disciplines. Il s'agit toutefois d'un cadre pédagogique a priori favorable au développement des sciences humaines et sociales, plus largement des « humanités », de par les recommandations de la CTI ; mais ce cadre peut aussi être compris comme finalisé et plus contraint qu'il n'y parait, suivant en cela assez étroitement l'organisation des écoles d'ingénieurs qui elle-même découle de leur histoire et de leurs traditions pédagogiques. Le premier article a permis de cerner la patiente genèse de deux grands courants dans la formation des élèves ingénieurs en France, l'un tourné vers le spécialiste l'autre vers le généraliste. Les deux études de cas confirment cette dualité, toujours puissamment à l'œuvre aujourd'hui, et elles l'éclairent par le récit du réel et du concret de deux expériences opposées.

Enseigner les sciences humaines et sociales en école d'ingénieur consiste d'abord à comprendre une culture d'école, de département même, puis à s'y insérer et à négocier son projet. Celui-ci doit 
réunir une équipe la plus large possible, pour faire simultanément des propositions précises d'unités de valeur et montrer une vision globale des besoins de l'école. La capacité à proposer une maquette pédagogique d'ensemble à Polytech Nantes, pour une formation équilibrée et adaptée aux besoins tant de l'école que des différents départements, a permis une avancée incontestable des « humanités », dans un contexte pourtant peu favorable. Une telle approche globale est une condition nécessaire pour se donner une chance de réussite conforme aux attentes de l'équipe, en contenus convenablement définis et en heures d'enseignement. Le projet individuel portant sur une seule discipline conduit à des situations plus incertaines en termes de résultat final et beaucoup plus difficile à pérenniser au cours du temps. Suite à nos expériences personnelles de négociation en tant que responsable d'une équipe SHS, dans deux écoles très différentes, le projet de définition de maquette ou de sa modification doit être proposé le plus en amont possible dans les processus de décision, et si possible au niveau de l'école dans son ensemble. C'est le seul moyen de lui permettre de dépasser le véritable écueil des besoins des départements de l'école qui, pris isolément, sont généralement réducteurs et destructeurs de sens, au nom de l'utilité pédagogique et de la sacro-sainte efficacité pratique des technologues.

Rappelons en conclusion que la nature des SHS en écoles d'ingénieurs, comme nous l'avons noté à différentes reprises dans nos deux articles de ce volume, posent un véritable problème de définition. Le point de départ de cette définition en école d'ingénieurs est souvent en creux : ce qui n'est pas $\mathrm{du}$ ressort des enseignements scientifiques et technologiques, ou bien à caractère utilitariste : ce qui renforce et complète l'efficacité du technoscientifique. Fort heureusement, quelques exemples montrent que des approches beaucoup plus positives existent, prenant si l'on peut dire les SHS au sérieux et comme discipline à part entière ; enfin un quatrième courant de leur définition peut être identifié dans la reconnaissance d'un besoin de culture générale et de formation à l'humanisme des élèves ingénieurs. La question épistémologique de la nature des sciences humaines et sociales en écoles d'ingénieurs, posée en introduction de cet article et en filigrane du premier demeure. S'agit-il de SHS spécifiques aux écoles ?, de SHS pour les ingénieurs, dans une direction que l'on pourrait qualifier de SHS appliquées au monde de l'entreprise et aux ingénieurs, en reprenant la terminologie courante des « sciences appliquées » ${ }^{15}$. En ce sens, les sciences de gestion, l'économie appliquée à l'entreprise, la sociologie du travail, l'éthique et le droit des affaires, l'ergonomie, l'histoire des ingénieurs ou l'histoire des techniques pourraient fort bien correspondre à ce type de définition des SHS, en voie de spécialisation « technologique » à leur tour, à la suite des sciences exactes. Ou s'agit-il d'une simple extension du domaine des SHS académiques classiques en direction d'objets spécifiques ? Nous ne trancherons pas, bien entendu, mais cette question doit être réfléchie pour toute recherche un peu approfondie dans le domaine. Des questions d'épistémologie des SHS sont soulevées, et elles sont inévitables ; tout comme il serait absurde de refuser l'entrée dans le monde des SHS, certes parfois problématique, qu'offrent les enseignements non scientifiques ou techniques en écoles d'ingénieurs, au nom de leur «impureté » native ou de leurs faiblesses de contenus ou de méthodes, et de les cantonner à de simples recettes pédagogiques. L'examen de ces questions renvoie là aux enseignements individuels, à la capacité de chaque enseignant-chercheur à situer les contenus de ses cours et ses pratiques pédagogiques au sein de sa propre discipline de sciences humaines et sociales et en relation avec sa propre recherche. Ce travail est à faire par chacun et nous l'avons esquissé pour une partie de nos pratiques pédagogiques, dans le domaine des maquettes numériques de machines anciennes, clairement à l'intersection de nos compétences en histoire des techniques et des outils numériques développés dans l'industrie de la conception mécanique. Un champ de recherche est incontestablement à développer là, invitant chacun à réfléchir au lien spécifique entretenu par ses enseignements avec les problématiques et les questionnements méthodologiques de son champ disciplinaire. La question épistémologique renvoie en définitive à des responsabilités scientifiques individuelles.

\footnotetext{
15 Les sciences appliquées renvoient au concept plus tardif, mais aujourd'hui commun, de « sciences pour l'ingénieur » ou SPI. Un certain parallèle peut s'établir, notamment en recherche, entre SHS et SPI.
} 
Une dernière remarque pratique à propos des questions de nature et de définition des SHS en écoles d'ingénieurs, de la part d'un ancien responsable du domaine des humanités en école d'ingénieurs : la réponse qui sera apportée dépend des types de postes mis au recrutement. Autrement dit, il s'agit de ne pas raisonner uniquement sur des contenus d'enseignements, par définition évolutifs et négociables, mais aussi sur les acteurs et sur leur profil scientifique. Il est important, de notre point de vue, qu'il s'agisse de postes d'enseignants chercheurs et non simplement de PRAG ${ }^{16}$ ou d'intervenants professionnels ${ }^{17}$, car la garantie scientifique du travail programmatique et pédagogique à effectuer se situe en premier lieu dans la recherche. C'est elle qui identifie et justifie l'intervention, qui apporte une approche critique solidement étayée et une problématisation qui dépasse le cadre purement utilitaire souvent posé au départ par l'institution. En ce sens, le rattachement à un laboratoire est essentiel, et l'une des tâches premières est d'organiser des structures de recherches simultanément validées par les instances académiques et ouvertes en réseau pour rattacher valablement des chercheurs isolés, comme c'est souvent le cas en écoles. Les créations de laboratoires spécifiques de SHS en écoles, ou d'équipes pluridisciplinaires à l'interface des SHS et des SPI, comme nous en avons cité quelques exemples (Mines Paris, UT Belfort-Montbéliard, Ecole centrale de Nantes), sont certes emblématiques mais condamnées à rester relativement peu nombreuses ou ponctuelles dans le contexte actuel d'évaluation et de financement de la recherche.

\section{Références}

Cotte, Michel, 2012. Documents pour l'histoire des techniques : La numérisation du patrimoine technique, éditeur du n ${ }^{\circ} 18$, Paris CNAM, $2^{\text {ème }}$ semestre 2009 ; «Les outils numériques au service de l'histoire des techniques », e-Phaïstos, vol. I-2 Paris, décembre, p. 12-27.

Didier, Christelle, 2008. Penser l'éthique des ingénieurs, Presses universitaires de France, Paris.

Lamard, Pierre, \& Lequin, Y.-C., 2005. La technologie entre à l'université. Compiègne, Sevenans, Belfort-Montbéliard, Belfort-Montbéliard, UTBM. Consulté à l'adresse http://www . lavoisier.fr/livre/notice . asp?ouvrage=2263207

\footnotetext{
16 Il s'agit d'une réserve sur le type de poste (double d'heures en présence des élèves par rapport à un maitre de conférences, pas d'obligation de recherche statutaire) et en aucun cas à propos des collègues PRAG qui sont généralement d'excellents pédagogues et qui, souvent, se conduisent à titre individuel comme des chercheurs en soutenant des thèses et en publiant.

${ }^{17} \mathrm{La}$ remarque précédente vaut également ici, en notant l'extrême diversité que peut recouvrir le terme d'intervenant professionnel.
} 